Europhys. Lett., 43 (6), pp. 719-724 (1998)

\title{
Clusters of synchronization and bistability in lattices of chaotic neurons
}

\author{
R. Huerta ${ }^{1,3}$, M. BAzHenov ${ }^{2}$ and M. I. Rabinovich ${ }^{3}$ \\ 1 Department of Computer Engineering, Universidad Autónoma de Madrid \\ Cantoblanco, 28049 Madrid, Spain \\ 2 Howard Hughes Medical Institute, The Salk Institute \\ Computational Neurobiology Laboratory, La Jolla, CA 92037, USA \\ 3 Institute for Nonlinear Science, University of California \\ San Diego, La Jolla, CA 92093-0402, USA
}

(received 12 February 1998; accepted in final form 17 July 1998)

PACS. 87.10+e - General, theoretical, and mathematical biophysics (including logic of biosystems, quantum biology, and relevant aspects of thermodynamics, information theory, cybernetics, and bionics).

PACS. 87.22As - General theory and phenomenology.

\begin{abstract}
We investigated different regimes of synchronization in large lattices of chaotic spiking-bursting neurons. The lattices exhibit the following features: developed spatio-temporal disorder with no synchronization; spatial clusters of bursting synchronization; homogeneous bursting synchronization; and complete chaotic synchronization. We observed a bistable synchronization phenomenon in a wide region of the control parameter space. The bistability exists for homogeneous bursting synchronization with long-range correlation and spatial clusters of partial synchronization. The bistable regime appears in lattices with a size larger than the space-scale of these clusters.
\end{abstract}

Introduction. - The analysis of the behavior of large assemblies of chaotic elements has been the subject of recent investigations, and is of interest both from the fundamental [1-5] and modeling points of view [6-9]. In particular, a network of chaotic elements is currently a very popular ingredient of information processing [7].

We report in this letter on the collective dynamics of chaotic neurons with local interactions. Recent work [3-6] indicates that such lattices often exhibit "non-trivial" cooperative behavior demonstrating a rich variety of phase transitions. Such behavior is "non-trivial" because the chaotic dynamics of these lattices with short-scale interaction must exhibit extensive chaos: the number of Lyapunov exponents increases with the size of the lattice (see, for example, $[1,2]$ ). Actually, the cooperative behavior of chaotic lattices depends strongly on the strength of the local connection, variations of which reveal a rich diversity of synchronization.

The model. - The lattice is made of non-identical Hindmarsh-Rose neurons [10] placed randomly inside the chaotic regime. Each element is electrically coupled to its nearest neighbors. 

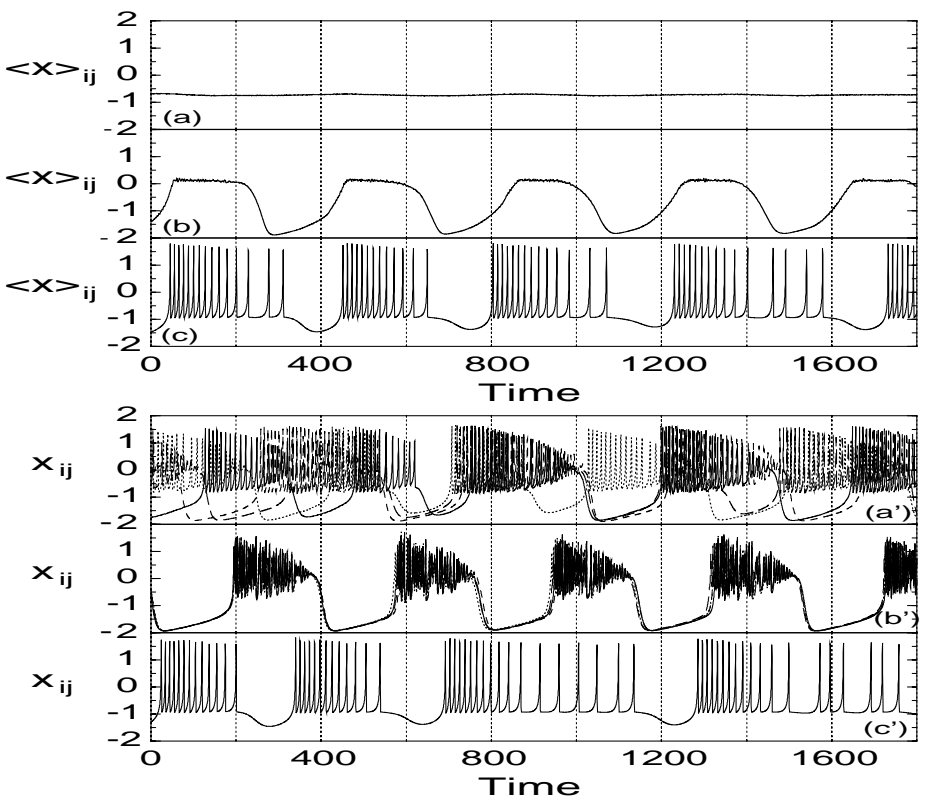

Fig. 1. - Average activity and membrane potential for a lattice of $100 \times 100$ Hindmarsh-Rose models with (a) $\epsilon=0.04$, (b) $\epsilon=0.4$, and (c) $\epsilon=230$. The membrane potential, $x$, of 4 different units chosen at random locations in the $100 \times 100$ lattice with $\left(\mathrm{a}^{\prime}\right) \epsilon=0.04,\left(\mathrm{~b}^{\prime}\right) \epsilon=0.4$, and $\left(\mathrm{c}^{\prime}\right) \epsilon=230$.

The system is described by the following set of coupled ordinary differential equations:

$$
\begin{aligned}
\frac{\mathrm{d} x_{i j}}{\mathrm{~d} t} & =y_{i j}+3 x_{i j}^{2}-x_{i j}^{3}-z_{i j}+I_{i j}-\epsilon\left(4 x_{i j}-x_{i+1, j}-x_{i-1, j}-x_{i, j+1}-x_{i, j-1}\right), \\
\frac{\mathrm{d} y_{i j}}{\mathrm{~d} t} & =1-5 x_{i j}^{2}-y_{i j}, \\
\frac{\mathrm{d} z_{i j}}{\mathrm{~d} t} & =-r z_{i j}+r S\left(x_{i j}+1.6\right),
\end{aligned}
$$

for which $i, j=1, \ldots, N, r=0.0021, I_{i j}=3.281 \pm 0.05$ and $S=4$. The modifiable parameter is $\epsilon$, the strength of the electrical coupling. For the periodic boundary conditions used in this investigation, the system was integrated using a Runge-Kutta 6(5) scheme with variable time step based on [11]. The absolute error was $10^{-15}$ and the relative error was $10^{-6}$ in all the calculations presented in this article. The code to integrate this lattice is available at http://inls.ucsd.edu/ huerta.

The dynamics of the Hindmarsh-Rose model is characterized by two very different oscillation time scales, as are living bursting neurons. The subsystem $\left(x_{i j}, y_{i j}\right)$ is responsible for the fast oscillations (the spikes) and the slow subsystem is carried by $z_{i j}$ and $x_{i j}$. The fast subsystem is placed nearby the homoclinic bifurcation and, together with the slow subsystem, generates chaotic oscillations $[12,13]$.

To study the global behavior of the lattice, besides the visual exploration of results, we use two quantities: the average activity of the lattice $\langle x(t)\rangle=\left(1 / N^{2}\right) \sum_{i, j=1}^{N} x_{i j}(t)$ to identify the 

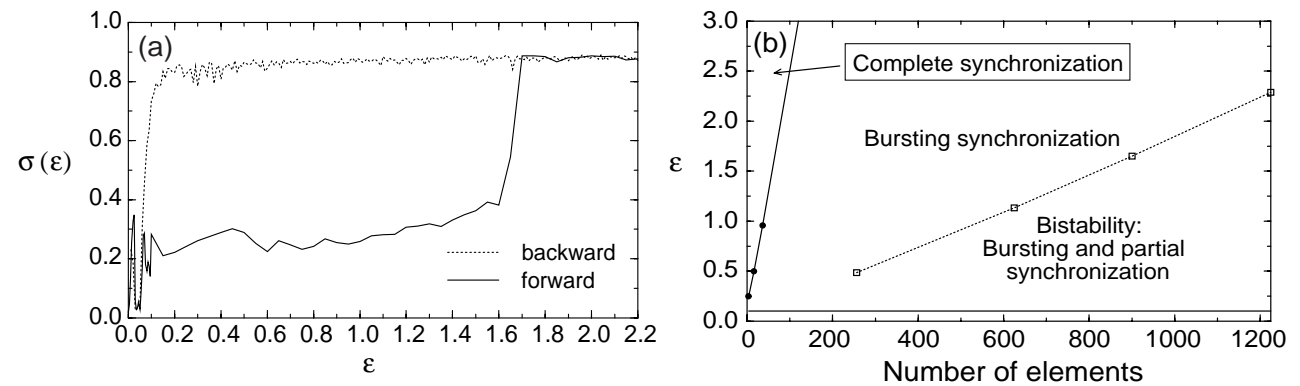

Fig. 2. - (a) Variance of the oscillations for decreasing values (dotted line) and increasing values (solid line) of $\epsilon$ for a lattice of $30 \times 30$ elements. (b) Bifurcation diagram between bursting and complete synchronization solid line. The dotted line indicates the upper boundary of bistability between partial synchronization and bursting synchronization. The lower line parallel to the $x$-axis indicates the boundary between no synchronization and the bistability region.

temporal characteristics; and the spatial correlation function

$$
C(r)=\left\langle\frac{1}{N_{\boldsymbol{\eta}(r)}} \sum_{\boldsymbol{\eta}(r)} \frac{\left(x \boldsymbol{\eta}(r)(t)-\langle x \boldsymbol{\eta}(r)\rangle_{t}\right)\left(x \boldsymbol{\eta}_{0}(t)-\left\langle x \boldsymbol{\eta}_{0}\right\rangle_{t}\right)}{\left(x \boldsymbol{\eta}_{0}(t)-\left\langle x \boldsymbol{\eta}_{0}\right\rangle_{t}\right)^{2}}\right\rangle_{t},
$$

with $\boldsymbol{\eta}(r)=\left\{(i, j):(i-N / 2)^{2}+(j-N / 2)^{2}=r^{2} ; i, j=1 \ldots N\right\}$ and $\boldsymbol{\eta}_{0}=(N / 2, N / 2)$, where $N_{\boldsymbol{\eta}(r)}$ is the number of points on the circle of radius $r$.

The average activity is a valid quantity to distinguish between the different types of global synchronization. In order to clarify the meaning of the average activity and to show how it may be used to distinguish between synchronized and unsynchronized states, let us consider a specific case when the oscillations in all units are close to periodic $x_{i j}(t) \approx f\left(t+\phi_{i j}\right)$, where $\phi_{i j} \in[0, T]$ are the time lags of the oscillators. The average activity of the simplified system is given by $\langle x(t)\rangle=\left(1 / N^{2}\right) \sum_{i, j=1}^{N} f\left(t+\phi_{i j}\right)$, which, in the limit $N \rightarrow \infty$ and for periodic $f(t)$, can be expressed as $\langle x(t)\rangle=\int_{0}^{T} p(\Phi) f(t+\Phi) \mathrm{d} \Phi$, where $p(\Phi)$ is the probability distribution of the time lags of the oscillators in the ensemble. When the elements are phase locked, $p(\Phi)=\delta\left(\Phi-\Phi_{0}\right)$, so they are completely synchronized, and $\langle x(t)\rangle=f(t)$. When the probability distribution is uniform in the interval $[0, T]$, then the ensemble is unsynchronized, and $\langle x(t)\rangle=(1 / T) \int_{0}^{T} f(\Phi) \mathrm{d} \Phi=$ constant.

Synchronization. - We found three main regimes of global synchronization: spiking (complete), partial (clustering), and bursting synchronization. In fig. 1 we plot the average activity for non-synchronization and for bursting and spiking synchronization for a layer of $100 \times 100$ elements. When there is no synchronization the average activity remains very close to a constant value as shown in fig. 1a. Figure 1 b gives a typical case of bursting synchronization where the average activity is periodic and nearly constant on top of each burst. This means that the spikes are completely unsynchronized. In fig. 1c an example of complete synchronization is displayed, where the behavior of one single unit is identical to the average activity of the whole lattice. Figure $1 a^{\prime}, b^{\prime}$ and $c^{\prime}$ show the time series of different units chosen at random locations in the lattice. The case of partial synchronization that will be explained in more detail in the following sections typically has an average activity with oscillations of an amplitude of 0.2 and the time series of nearby units are similar to fig. $1 b^{\prime}$, whereas for far away neurons the time traces are uncorrelated as in fig. $1 \mathrm{a}^{\prime}$. 


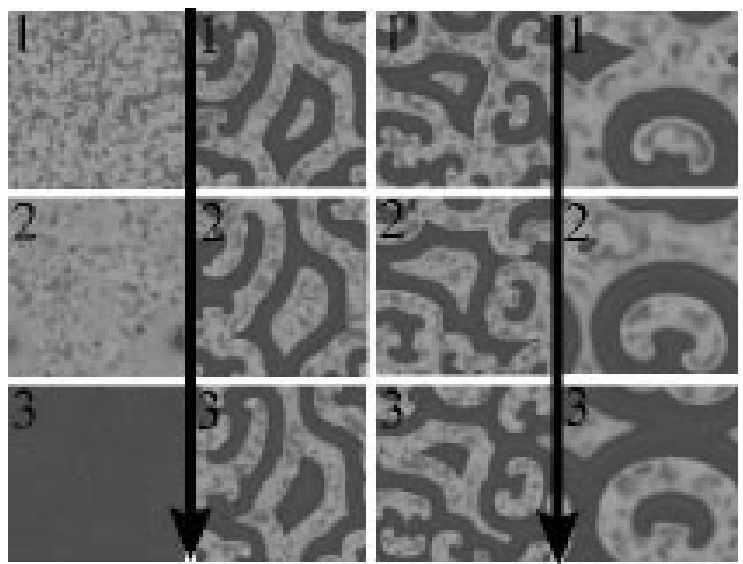

Fig. 3. - First column: three snapshots of the bursting synchronization regime in a $100 \times 100$ lattice for $\epsilon=0.5$. The first snapshot shows the beginning of the burst where the turbulent behavior is carried by the spiking activity. The second one shows the end of the burst where there are less spikes fired. And, finally, the third one represent the latency state at the bottom of the bursts. Second and third column: two different patterns, which are periodic on time, in the partial synchronization regime for $\epsilon=0.5$. Fourth column: partial synchronization for $\epsilon=1.5$. The solid arrows indicate the direction of time.

Bifurcations and bistability. - The quantity that we will use to discriminate between the different types of synchronization will be the average activity oscillation amplitude given by

$$
\sigma^{2}(\epsilon)=\frac{1}{T} \int_{0}^{T}\left(\left\langle\left\langle x_{\epsilon}\right\rangle_{i j}\right\rangle_{t}-\left\langle x_{\epsilon}(t)\right\rangle_{i j}\right)^{2} \mathrm{~d} t
$$

where $\left\langle x_{\epsilon}(t)\right\rangle_{i j}$ is the average activity of the lattice for a given value of the electrical connection $\epsilon$. In fig. 2a we plot $\sigma(\epsilon) v s$. the strength of the coupling for a lattice of $30 \times 30$ elements. Two regions can be identified: one of partial (cluster) synchronization with $\sigma(\epsilon)$ values ranging from 0.2 to 0.3 , and another with bursting synchronization with larger oscillations (see fig. 1). The region in which we are mainly interested is the bistable region where partial synchronization and bursting synchronization coexist. This region can be identified by the presence of the hysteresis loop for increasing and decreasing values of $\epsilon$. The magnitude of the pulsations of the average activity provide a means for differentiating between different regimes of global and partial synchronization.

How is the synchronization affected by the increasing size of the lattice? Figure $2 \mathrm{~b}$ exhibits the values for which the bifurcation from bursting to complete synchronization takes place. It has a strong linear dependence on the size of the lattice, $\epsilon_{c}=\alpha N^{2}$ with $\alpha=0.0247 \pm 10^{-4}$, which was fitted till at least a lattice of $100 \times 100$ (data not shown). The most interesting region is the bistable one where partial synchronization and bursting synchronization coexist as shown in fig. 2. The dotted line in fig. $2 \mathrm{~b}$ indicating the upper boundary of bistability has a strong linear dependence on lattice size (like complete bifurcation between bursting and complete synchronization) and the solid line parallel to the $x$-axis shows the lower boundary between the bistable region and no-synchronization. This lower bifurcation line exhibits only a very weak dependence on the size of the lattice. It is remarkable that the bifurcation point from bursting synchronization to non-synchronization is not a scalable property of the lattice.

A few snapshots of the activity of a lattice of $100 \times 100$ elements in the bistable region are given in fig. 3. The first column has a sequence of three snapshots corresponding to a bursting 


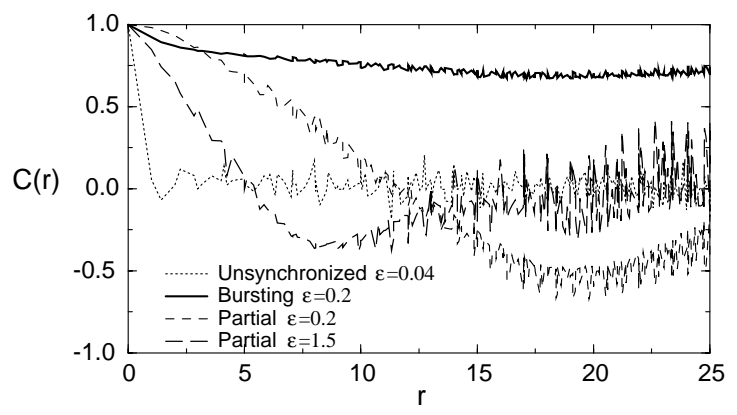

Fig. 4. - Spatial correlation calculated in a lattice of $70 \times 70$ elements for an unsynchronized state (dotted line), bursting synchronization (solid thick line), and partial synchronization for $\epsilon=0.2$ and $\epsilon=1.5$.

synchronization, where the spikes are not synchronized. In the second and third columns we present two different periodic patterns for different initial conditions corresponding to partial synchronization. These oscillating patterns are stable and have a characteristic space scale from 10 to 20 elements. The volume of the boundary of attraction for these periodic patterns is very small. The best way to reach them is moving $\epsilon$ slowly from 0 to any point in the region of bistability. The right column of fig. 3 shows another periodic pattern for $\epsilon=1.5$. Increasing the coupling strength between the elements leads to the growth of the space scale in the lattice. In fig. 4 the spatial correlation that distinguishes between non-synchronization, bursting, and partial (cluster) synchronization is presented. The space scale for partial synchronization is of the order of 10 elements. This explains why bistability for lattices smaller than $16 \times 16$ has not been observed heretofore. The clusters of partial synchronization with periodic synchronization is reminiscent of spatio-temporal patterns on the lattices of coupled periodic oscillators: the discrete variant of the Ginzburg-Landau model [14].

Conclusion. - In spite of the heterogeneous and chaotic nature of the individual elements, the lattices of spiking-bursting neurons coupled diffusively reveal unexpected spatio-temporal patterns: clusters of synchronization of slow dynamics. The boundaries of the clusters are the fronts of phase triggering between in-phase and out-of phase synchronization. These contrasting patterns reproduce themselves periodically in time. This regime coexists, in control parameter space, within the regime of bursting synchronization that is homogeneous in space and periodic in time. Depending on the initial conditions, one of these regimes is realized.

The presence of different patterns of synchronization for different initial conditions illustrates the rich possibility of this lattice to store the information coded in snapshots. The next feature to understand is how different patterns are loaded and retrieved as a function of external input and local interconnectivity. In spite of the obvious fact that the present model is an oversimplified description of the cooperative behavior of real cortex neural assemblies, bistability and cluster synchronization must be an important aspect of more complex networks with finite-scale interactions. It is important to notice that similar patterns have been reported in vivo in experiments with turtles using optical images [15], hallucinations [16], and models of cortical tissue [17]. The latest detailed investigation can be found in [18].

We want to acknowledge P. Weidman, L. Tsimring, H. Abarbanel, R. Llinas, V. Makarenko, J. J. Torres and P. Varona for fruitful and suggestive discussions. This work has been supported by grant DE-FG03-96ER14592. 


\section{REFERENCES}

[1] Kaneko K., Physica D, 23 (1986) 436.

[2] Egolf D. A. and Greenside H. S., Nature, 369 (1994) 851.

[3] Aranson I. S., Golomb D. and Sompolinsky H., Phys. Rev. Lett., 68 (1992) 3495.

[4] Brunnet L., Chaté H. and Manneville P., Physica D, 78 (1994) 141.

[5] Chaté H., Lemaitre A., Marq Ph. and Manneville P., Physica A, 224 (1996) 447.

[6] Gaponov-Grekhov A. V. and Rabinovich M. I., Chaos, 6 (1996) 259.

[7] Ishi S., Fukumizu K. and Watanabe S., Proceedings of the International Joint Conference on Neural Networks, Nagoya (Ibaraki, Japan) 1993.

[8] Hansel D. and Sompolinsky H., Phys. Rev. Lett., 68 (1992) 718.

[9] Hansel D. and Sompolinsky H., J. Comput. Neurosci., 3 (1996) 7.

[10] Hindmarsh J. L. and Rose R. M., Proc. R. Soc. London, Ser. B, 221 (1984) 87.

[11] Hull T. E., Enright W. H., Fellen B. F. and Sedgwick R. E., SiAM J. Num. Anal., 9 (1972) 603.

[12] Bazhenov M., Huerta R., Rabinovich M. I. and Sejnowski T., Physica D, 116 (1998) 392.

[13] Wang X.-J., Physica D, 62 (1993) 263.

[14] Gaponov-Grekhov A. V. and Rabinovich M. I., Nonlinearity in Action: Oscillation (Springer, Berlin) 1992.

[15] Prechtl J. C., Cohen L. B., Pesaran B., Mitra P. P. and Kleinfeld D., Proc. Natl. Acad. Sci. (USA), 94 (1997) 7621.

[16] Ermentrout G. B. and Cowan J. D., Biol. Cybern., 34 (1979) 137.

[17] C. Fohlmeister, W. Gerstner, R. Ritz and J. L. van Hemmen, Neural Comput., 7 (1995) 1046.

[18] W. M. Kistler, R. Seitz and J. Leo van Hemmen, Physica D, 114 (1998) 273. 